\title{
One Zone Numerical Model for the Galactic Evolution of Lithium
}

\author{
Marco Terra ${ }^{1}$ and Lilia I. Arany-Prado \\ Observatório do Valongo/Universidade Federal do Rio de Janeiro
}

\begin{abstract}
We analyze the evolution of ${ }^{7} \mathrm{Li} / \mathrm{H}$ using the basic model for the galactic chemical evolution (GCE) described by Brown (1992) and a numerical procedure which allows to estimate the contribution of the red giants to the enrichment of lithium in the interstellar medium (ISM).
\end{abstract}

\section{Motivation and Models}

Since the discovery of the Li rich giants, stellar nucleosynthesis has been regarded as an important source for the galactic $\mathrm{Li}$ in conjunction with cosmic rays spallation and the Big Bang primordial nucleosynthesis. Also low mass stars represented by $\mathrm{K}$ giants became a promising source of Li (de la Reza et al. 1996; Gregorio-Hettem et al. 1993). Our goal is to evaluate the contribution of the red giants to the enrichment of $\mathrm{Li}$ in the ISM. Our numerical procedure considers the one zone GCE model, assumes the sudden mass loss approximation; the IMF and the turnoff mass of Scalo (1986); two different star formation rates (SFRs) (figure 1a): the one of Mathews \& Schramm (M\&S, see Brown 1992) and the maximum exponentially decreasing (ExpD, Miller \& Scalo 1979). The mass of the remnants is taken from Iben \& Renzini (1983). The galactic disk is supposed to be 12 Gyr old. We use the following mass ranges: $1-2.5 \mathrm{M}_{\odot}$ for red giants; $2.5-7 \mathrm{M}_{\odot}$ for AGB stars; and $7-62 \mathrm{M}_{\odot}$ for supernovae $(\mathrm{SNe})$.

\section{Results and Comments}

We establish the return mass fraction to the ISM, R(t) (Arany-Prado \& Maciel, 1998), for each SFR (figure 1b). The numerical procedure allows to analyze each site separately. We assume as the maximum values for the lithium abundance in the stellar ejecta: $10^{-8}$ for AGB stars (Smith \& Lambert 1990) and $10^{-10}$ for $\mathrm{SNe}$ (Brown et al. 1990). If we consider: a) the maximum Li abundance ejected by $\mathrm{K}$ giants as $2 \times 10^{-8}$ (Terra et al. 2000); b) the observational remark of Brown et al. (1989) that near $2 \%$ of red giants are Li rich; c) the evolutionary argument from de la Reza et al. (1996), we conclude that the mean value for the lithium abundance ejected at any short time range by $\mathrm{K}$ giants is up to $4 \times 10^{-9}$. Taking into account the contribution to the ISM due to stellar sites plus cosmic ray spallation, the mean $\mathrm{Li}$ abundance in the red giants ejecta has to be about $3 \times 10^{-9}$ in order to total $\mathrm{Li}$ fit the present abservational constraints. This is in good agreement with the arguments above. Furthermore, $\mathrm{K}$ giants become the most important site for Li production at about the fourth Gyr of the galactic 

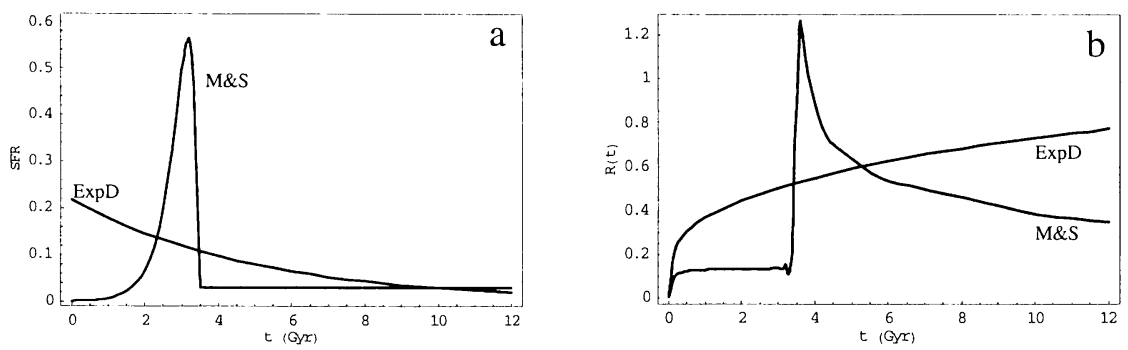

Figure 1. a) the two SFRs. b) $R(t)$ for each SFR.
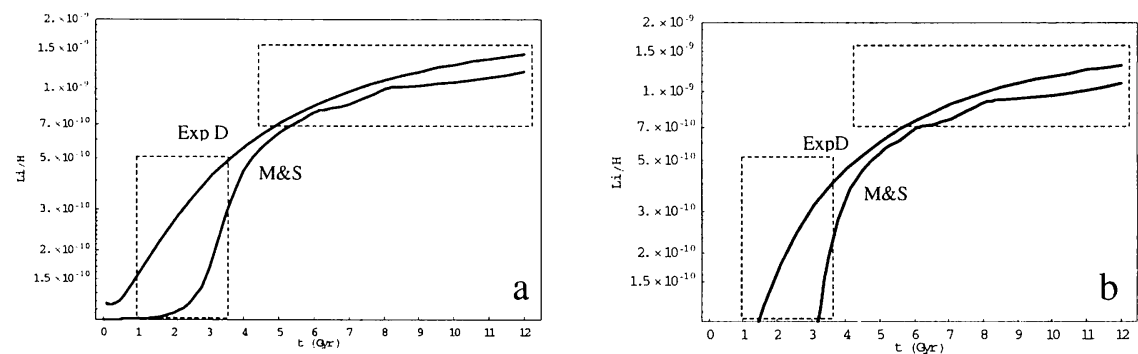

Figure 2. The evolution of ${ }^{7} \mathrm{Li} / \mathrm{H}$ with time in the ISM considering two SFRs and the following: a) with Big Bang nucleosynthesis; b) only stellar nucleosynthesis. Boxes represent the observational constraints given by Brown (1992).

life. We show the results of our numerical models considering the primordial nucleosysnthesis (figure 2a) and also the arguments of Burbidge \& Hoyle (1998), for whom only stellar nucleosynthesis is responsible for the elements generation (figure 2b).

Terra, M. would like to thank FAPERJ for financial support: project 150.571/98

\section{References}

Arany-Prado, L.I., Maciel, W., 1998, Rev.Mex.Ast.Astrof., 34, 21

Brown, L.E., 1992, ApJ, 389, 251

Brown, J.A., Sneden, C., Lambert, D.L., Dutchover, E.J., 1989, ApJS, 71, 293

Brown, L.E., Dearborn, D.S., Schramm, D.N., Larsen, J.T., Kurokawa, S., 1990, ApJ, 371, 648

Burbidge, G., Hoyle, F., 1998, ApJ, 509, L1

de la Reza, R., Drake, N., da Silva, L., 1996, ApJ, 456, L115

Gregorio-Hetem, J., Castilho, B.V., Barbuy, B., 1993, A\&A, 268, L25

Iben, I., Renzini, A., 1983, ARA\&A, 21, 271

Miller, G.E., Scalo, J.M. 1979, ApJS, 41, 513

Scalo, J M , 1986, Fund. Cosmic Phys., 11, 1

Smith, V., Lambert, D., 1990, ApJ, 361, L69

Terra, M., de la Reza, R., Batalha, C., 2000, in preparation 